\title{
Efeito do sítio de venopunção nos parâmetros hematológicos em tigre-d'água-americano, Trachemys scripta elegans ${ }^{1}$
}

\author{
Nina C. Medeiros ${ }^{2 *}$, Rosangela Locatelli-Dittrich ${ }^{3}$, Elizabeth M.S. Schmidt ${ }^{4}$, Alessandra \\ A.A. Alvares ${ }^{5}$, Lia L. Patrício ${ }^{2}$, Rogério R. Lange ${ }^{3}$ e Rodrigo A.M. de Souza ${ }^{6}$
}

\begin{abstract}
Medeiros N.C., Locatelli-Dittrich R., Schmidt E.M.S., Alvares A.A.A., Patrício L.L., Lange R.R. \& Souza R.A.M. 2012. [Effect of the blood collection site on the hematological parameters in red-eared slider, Trachemys scripta elegans.] Efeito do sítio de venopunção nos parâmetros hematológicos em tigre-d'água-americano, Trachemys scripta elegans. Pesquisa Veterinária Brasileira 32(Supl.1):37-40. Laboratório de Patologia Clínica Veterinária, Departamento de Medicina Veterinária, Universidade Federal do Paraná, Rua dos Funcionários 1540, Curitiba, PR 80035-050, Brazil. E-mail: ninadcm@hotmail.com

The aim of the present study was to compare two different blood collection sites of 24 red-eared sliders (Trachemys scripta elegans) from a commercial breeder, situated in Antonina, Paraná, Brasil. The animals were physically restrained and paired blood samples were collected from the dorsal coccygeal vein and the occipital sinus. The samples were collected in syringes containing heparin. After collection the samples were identified and refrigerated to posterior hematological analysis. Red blood cell and white blood count were performed using a hemocytometer. The packed cell volume and hemoglobin were determined by the microhematocrit method and cyanmethemoglobin method, respectively. Total plasma protein was determined by refractometry and differential leukocyte count was performed by the Shilling technique. There was a significant difference in the total white blood cell count and on the total plasma protein value, and on both cases the results found on the samples from the dorsal coccygeal vein were lower. The difference found on the white blood cell count was probably due to the lymph contamination of the samples, which also justifies the lower values of total plasma protein. In conclusion, the occipital sinus is more recommended blood collection site than the dorsal coccygeal vein.
\end{abstract}

INDEX TERMS: Trachemys scripta, red-eared slider, blood collection, dorsal coccygeal vein.

RESUMO.- 0 objetivo do presente estudo foi realizar a comparação entre dois sítios de coleta sanguínea em 24 exemplares de tigre-d'água-americano (Trachemys scripta ele-

\footnotetext{
${ }^{1}$ Recebido em 6 de julho de 2012.

Aceito para publicação em 8 de outubro de 2012.

${ }^{2}$ Médica Veterinária Autônoma, Rua José Ananias Mauad 413, Curitiba, PR 80210-130, Brasil.*Autor para correspondência: ninadcm85@gmail.com

${ }^{3}$ Departamento de Medicina Veterinária, Universidade Federal do Paraná (UFPR), Rua dos Funcionários 1540, Curitiba, PR 80035-050. E-mail: roslocdi@ufpr.br

${ }^{4}$ Depto Clínica Veterinária, Faculdade de Medicina Veterinária e Zootecnia, Universidade Estadual Paulista (Unesp), Campus Botucatu, Distrito de Rubião Junior s/n, Botucatu, SP 18618-970, Brasil. E-mail: bethschmidt@fmvz.unesp.br

${ }_{5}^{5}$ Departamento de Medicina Veterinária, Hospital Veterinário Centro Universitário de Maringá, Av. Guedner 1610, Maringá, PR 87050 390, Brasil. E-mail: ale_alvares@yahoo.com.br

${ }^{6}$ Departamento de Medicina Veterinária, Universidade Estadual do Centro-Oeste, Rua Camargo Varela de Sá 3, Guarapuava, PR 85040-080, Brasil. E-mail: rodrigo.unicentro@gmail.com
}

gans) oriundos de um criadouro comercial, localizado no município de Antonina, litoral do Paraná, Brasil. Os animais foram submetidos a contenção física e as venopunções foram realizadas no seio supraocciptal e na veia coccígea dorsal. As amostras heparinizadas foram identificadas e refrigeradas para posterior análise laboratorial. A contagem total de eritrócitos e leucócitos foi realizada pela técnica de hemocitometria. $\mathrm{O}$ hematócrito $(\mathrm{Ht})$ e a hemoglobina $(\mathrm{Hb})$ foram determinados pelo método de microhematócrito e cianometahemoglobina, respectivamente. A proteína plasmática total (PPT) foi determinada por refratometria e a contagem diferencial de leucócitos foi realizada através da técnica de Shilling. Houve diferença significativa no número de leucócitos e no valor da proteína plasmática total, e em ambos os casos os valores encontrados nas amostras provenientes da veia coccígea dorsal foram inferiores. A diferença encontrada no número de leucócitos provavelmente foi devido à contaminação por linfa, que também justifi- 
ca o menor valor na concentração da proteína plasmática total. Conclui-se que é mais indicada a venopunção no seio supraocciptal quando comparado a veia coccígea dorsal.

TERMOS DE INDEXAÇÃO: Trachemys scripta, tigre-d água-americano, venopunção, seio venoso supraoccipital, veia coccígea dorsal.

\section{INTRODUÇÃO}

O tigre d'água americano (Trachemys scripta elegans) é uma espécie de cágado de água doce que pertence a ordem testudines, subordem Cryptodira, família Emydidae e subfamília Emydinae (Ernst \& Barbour 1989). São animais que originariamente habitavam os Estados Unidos da América até o Norte da América do Sul, porém, atualmente é considerada espécie invasora no Brasil. Estão introduzidos em nosso meio ambiente e também são criados como animais de estimação. Devido à introdução desses animais na fauna brasileira e sua crescente participação como "pet" exótico, torna-se necessário conhecer o perfil hematológico desta espécie para acessar o estado fisiológico dos animais, além dos fatores extrínsecos e intrínsecos que podem alterar esses valores.

Na medicina de animais selvagens, os exames laboratoriais são ferramentas utilizadas para diagnóstico de doenças e como biomarcadores de agressões ambientais, uma vez que a sanidade do meio ambiente influencia na vida dos seres que interagem com esse (Almosny \& Monteiro 2006).

Na avaliação dos parâmetros hematológicos é necessário interpretar e analisar se as diferenças encontradas são significativas, pois fatores externos e internos podem interferir nos resultados. A espécie em questão, o estado de saúde do animal, a idade, o sexo, o estado fisiológico e reprodutivo são os fatores intrínsecos mais importantes. Por outro lado, o sítio de coleta de sangue, temperatura, estação do ano e o ambiente no qual o animal se encontra são fatores extrínsecos que alteram os resultados hematológicos (López-Olvera et al. 2003, Sykes \& Klaphake 2008).

Os principais locais de venopunção em quelônios são: veia jugular, punção cardíaca, veia coccígea dorsal. Outros sítios são descritos (plexo venoso braquial ou femoral, seio orbital, seio venoso supraoccipital), mas são considerados de menor valor clínico devido à maior possibilidade de contaminação por linfa (Samour et al. 1984, Jacobson 1993, Dyer \& Cervasio 2008). A escolha do sítio de coleta de sangue depende de diversos fatores como a espécie, tamanho do indivíduo, localização anatômica das veias superficiais, sexo e idade, volume a ser coletado e habilidade do clínico veterinário (Samour et al. 1984, Murrey 2000).

Desta forma, o local de coleta da amostra é um fator importante a ser considerado em répteis. Estudos foram realizados por López-Olvera et al. (2003) em Testudo marginata, por Cuadrado et al. (2003) em Chamaeleo chamaeleon e por Gottdenker \& Jacobson (1995) em Gopherus agassizii, entretanto, até o presente, os efeitos dos diferentes sítios de coleta de sangue nos parâmetros hematológicos de tigre-d’água-americano não foram estabelecidos.

0 objetivo do presente estudo foi analisar o efeito dos sítios de coleta, seio venoso supraoccipital e veia coccígea dorsal, nos parâmetros hematológicos em tigre-d`água- -americano (Trachemys scripta elegans). 0 estudo objetiva também contribuir com os valores hematológicos para o tigre-d'água americano devido a escassez de parâmetros de referência para essa espécie.

\section{MATERIAL E MÉTODOS}

Foram realizadas coletas de sangue de 24 exemplares de tigre-d'água-americano (Trachemys scripta elegans) pertencentes a um criadouro comercial localizado no município de Antonina, litoral do Paraná (Registro no IBAMA 2682286). Os animais estavam alojados em um tanque de água sem aquecimento. A temperatura média registrada na cidade de Antonina no dia da coleta foi de $22,5^{\circ} \mathrm{C}$, com temperatura mínima de $16,9^{\circ} \mathrm{C}$ (Paraná 2004). Os tigres-d'água foram submetidos a contenção física e subseqüente coleta de sangue. De cada indivíduo, foi realizada coleta em dois locais distintos: seio venoso supraocciptal (localizado dorsalmente na região cervical, caudalmente ao osso occipital) e veia coccígea dorsal (na cauda). Foram utilizadas seringas heparinizadas e o volume de $1,0 \mathrm{ml}$ de sangue foi coletado por local de venopunção. Após a coleta, as amostras foram devidamente identificadas e armazenadas sob refrigeração para posterior análise. As extensões sanguíneas foram realizadas imediatamente após a coleta de sangue. As análises foram realizadas no Laboratório de Patologia Clínica Veterinária da Universidade Federal do Paraná.

Para a determinação do hematócrito foi utilizado o método do microhematócrito e a concentração de hemoglobina foi determinada pelo método da cianometahemoglobina, com centrifugação (Raskin 2000, Jain 2010).

As contagens totais de eritrócitos e leucócitos foram realizadas em câmara de Neubauer, com diluição do sangue a 1:100 em solução de Azul de Cresil Brilhante. As extensões sanguíneas foram coradas com corante hematológico de Wright para a contagem diferencial de leucócitos (100 células). As concentrações das proteínas plasmáticas totais foram determinadas por refratometria (Jain 2010).

Os parâmetros foram determinados e organizados em dois grupos (seio venoso supraocciptal e veia coccígea dorsal) e comparados no programa estatístico StatView ${ }^{\circledR}$.

\section{RESULTADOS E DISCUSSÃO}

Os valores hematológicos obtidos nos dois locais de coleta de sangue estão apresentados no Quadro 1.

Verificou-se diferença significativa $(\mathrm{p}<0,05)$ na conta-

Quadro 1. Parâmetros hematológicos de tigre-d'água-americano (Trachemys scripta elegans) em dois diferentes sítios de coleta

\begin{tabular}{|c|c|c|c|c|}
\hline \multirow[t]{2}{*}{ Parâmetros } & \multicolumn{2}{|c|}{$\begin{array}{l}\text { Seio venoso } \\
\text { supraocciptal }\end{array}$} & \multicolumn{2}{|c|}{$\begin{array}{c}\text { Veia coccígea } \\
\text { dorsal }\end{array}$} \\
\hline & $\begin{array}{l}\text { Mínimo- } \\
\text { Máximo }\end{array}$ & Média & $\begin{array}{l}\text { Mínimo- } \\
\text { Máximo }\end{array}$ & Média \\
\hline Eritrócitos totais $\left(\mathrm{X} 10^{6} / \mu \mathrm{L}\right)$ & $3,8-6,8$ & 5,3 & $3,1-6,5$ & 4,8 \\
\hline Leucócitos totais $(/ \mu \mathrm{L})$ & $6.612-21.054$ & $13.833_{\mathrm{a}}$ & $5.692-13.724$ & $9.708_{b}$ \\
\hline Hemoglobina (g/dl) & $5,2-9,0$ & $7,1_{a}$ & $4,3-10,1$ & 7,2 \\
\hline Hematócrito (\%) & $12-24$ & $18^{\mathrm{a}}$ & $13-23$ & $18^{\mathrm{a}}$ \\
\hline Heterófilos (\%) & $31-53$ & $42_{\mathrm{a}}$ & $27-51$ & $399^{\circ}$ \\
\hline Linfócitos (\%) & $5-15$ & $10^{d}$ & $5-17$ & $11_{a}^{a}$ \\
\hline Eosinófilos (\%) & $27-47$ & $37_{a}^{a}$ & $25-53$ & $39^{a}$ \\
\hline Basófilos (\%) & $1-9$ & $4 a$ & $1-7$ & $4 a^{a}$ \\
\hline Monócitos (\%) & $4-13$ & $9^{a}$ & $3-15$ & 9 \\
\hline PPT (g/dl) ${ }^{\mathrm{a}}$ & $1,8-4,4$ & $3,1_{a}^{d}$ & $1,3-3,3$ & $2,3_{b}$ \\
\hline
\end{tabular}

$\overline{\mathrm{PPT}}=$ Proteína plasmática total. Letras diferentes na mesma linha significam diferença significativa $(\mathrm{p}<0,05)$. 
gem total de leucócitos e nas concentrações de proteínas plasmáticas totais. A contagem média total de leucócitos e as concentrações das proteínas plasmáticas totais foram superiores nas amostras obtidas do seio venoso supraocciptal. A contagem total de eritrócitos, a concentração de hemoglobina, o hematócrito e a contagem diferencial de leucócitos não apresentaram diferença entre os dois locais de venopunção.

Em jabutis-do-deserto (Gopherus agassizii), foram encontrados valores significativamente inferiores nas contagens totais de leucócitos, hematócrito, contagem total de eritrócitos e concentração de hemoglobina, para as amostras obtidas por punção do seio venoso supraoccipital quando comparados com os valores obtidos por venopunção da jugular (Gottdenker \& Jacobson 1995). De acordo com estes autores, a contaminação por linfa foi a causa dessas alterações. A diluição da amostra sanguínea com fluidos extravasculares e secreções podem alterar a composição do plasma, afetando os componentes celulares (Jacobson 1993, Murrey 2000).

López-Olvera et al. (2003) encontraram diferenças significativas para a concentração das proteínas séricas totais, contagem total de eritrócitos, hematócrito e hemoglobina entre amostras sanguíneas obtidas por punção da veia coccígea dorsal e por punção do plexo venoso braquial em jabutis da espécie Testudo marginata. De forma semelhante à observada no presente estudo, os autores supracitados reportaram valores inferiores para a concentração de proteínas totais nas amostras coletadas pela veia coccígea dorsal.

As diferenças encontradas no presente estudo entre o seio venoso supraoccipital e a veia coccígea dorsal, para a contagem total de leucócitos e concentração de proteínas plasmáticas, podem ter sido causadas pela contaminação por linfa da amostra sanguínea obtida por punção da veia coccígea dorsal, pois, segundo Jacobson et al (1992) a concentração de proteína total e de células é menor na linfa do que no soro. Os répteis, inclusive os quelônios, apresentam extensos e desenvolvidos vasos e seios linfáticos que são adjacentes ao sistema venoso, sugere-se que as diferenças nos parâmetros sanguíneos sejam causadas pela hemodiluição com linfa quando o sangue é obtido de vasos que não são visualizados no momento da coleta (Gottdenker \& Jacobson 1995).

Por outro lado, Cuadrado et al. (2003) realizaram comparação entre diferentes sítios de coleta em camaleão (Chamaeleo chamaeleon) e não encontraram diferenças significativas entre amostras obtidas por punção na veia coccígea ventral e veia jugular.

A temperatura é um fator que deve ser também considerado na avaliação das amostras sanguíneas obtidas de diferentes sítios de coleta. Sob baixas temperaturas ocorrem alterações cardiovasculares para a manutenção da temperatura corporal. Essas alterações incluem vasoconstrição dos vasos sanguíneos periféricos (Lillywhite et al. 1995, Murrey 2006). A temperatura da água em que os indivíduos avaliados no presente estudo estavam alojados foi de $20^{\circ} \mathrm{C}$. De acordo com Boyer \& Boyer (2006), a temperatura ideal da água para tartarugas aquáticas e semi aquáticas é de $25-37^{\circ} \mathrm{C}$. Desta maneira, sugere-se que as diferenças ob- servadas na contagem de leucócitos totais e nas concentrações das proteínas plasmáticas totais entre os dois locais de coleta ocorreram porque apesar do seio supraoccipital ser considerado um local de maior probabilidade à contaminação, sob baixas temperaturas, a veia coccígea dorsal é mais susceptível a vasoconstrição e, consequentemente, a amostra de sangue coletada neste sítio torna-se mais propícia a hemodiluição pela linfa.

No presente estudo verificou-se contaminação por linfa nas amostras obtidas da veia coccígea dorsal, de modo similar ao verificado por Gottdenker \& Jacobson (1995), em tartaruga do deserto e por López-Olvera et al (2003) em tartarugas da espécie Testudo marginata; entretanto, estes últimos autors não encontraram diferença significativa no número total de leucócitos, na temperatura ambiente próxima ao do presente estudo $\left(20,6^{\circ} \mathrm{C}\right)$.

Os resultados obtidos no presente estudo indicam que o local de venopunção, a temperatura ambiente e da água influenciam o número de leucócitos e a concentração de proteína plasmática total em Trachemys scripta elegans. Os parâmetros sanguíneos devem ser interpretados considerando o local de venopunção.

\section{CONCLUSÕES}

Os parâmetros eritrocitários obtidos nos dois locais de venopunção, seio venoso supraocciptal e veia coccígea dorsal, são similares e sem variação significativa.

A contagem total de leucócitos e as concentrações de proteínas plasmáticas totais são significativamente inferiores nas amostras de sangue obtidas pela punção da veia coccígea dorsal.

A hemodiluição pela contaminação por linfa foi a provável causa das alterações destes parâmetros em Trachemys scripta elegans mantidas em temperatura considerada inferior a ideal para cágados de água-doce em cativeiro.

A punção do seio venoso supraoccipital é mais indicada se comparada com a punção da veia coccígea dorsal para os animais mantidos sob baixas temperaturas.

\section{REFERÊNCIAS}

Almosny N.R. \& Monteiro A.0. 2006. Patologia clínica, p.939-966. In: Cubas Z.S. (Ed.), Tratado de Animais Selvagens: medicina veterinária. Roca, São Paulo.

Boyer T.H. \& Boyer D.M. 2006. Turtles, tortoises, and terrapins, p.78-99. In: Mader D.R. (Ed.), Reptile Medicine and Surgery. $2^{\text {nd }}$ ed. SaundersElsevier, St Louis.

Campbell T.W. 2006. Clinical pathology of reptiles, p.453-470. In: Mader D.R. (Ed.), Reptile Medicine and Surgery. $2^{\text {nd }}$ ed. Saunders-Elsevier, St Louis.

Cuadrado M., Molina-Prescott I. \& Flores L. 2003. Comparison between tail and jugular venipuncture techniques for blood sample collection in common chameleons (Chamaeleo chamaeleon). Vet. J. 166:93-97.

Dyer S.M. \& Cervasio E.L. 2008. An overview of restraint and blood collection techniques in exotic pet practice. Vet. Clin. Exotic. Pet. Pract. 11:423-443.

Ernst C.H. \& Barbour R.W. 1989. Turtles of the World. Smithsonian Institution Press, Washington, DC. 313p.

Gottdenker N.L. \& Jacobson E.R. 1995. Effect of venipuncture sites on hematologic and clinical biochemical values in desert tortoises (Gopherus agassizii). Am. J. Vet. Res. 56(1):19-21. 
Goulart C.E.S. 2004. Herpetologia, Herpetoultura e Medicina de Répteis. L.F. Livros de Veterinária, Rio de Janeiro, p.140-141.

Jacobson E.R., Schumacher J. \& Green M. 1992. Field and clinical techniques for sampling and handling blood for hematologic and selected biochemical determinations in desert tortoise, Xerobates agassizii. Copeia 1:237-241.

Jacobson E.R. 1993. Blood collection techniques in reptiles: laboratory investigations, p.144-152. In: Fowler M.E. (Ed.), Zoo and Wild Animal Medicine: Current therapy. $3^{\text {rd }}$ ed. W.B. Saunders, Philadelphia.

Jain N.C. 2010. Schalm's Veterinary Hematology. $6^{\text {th }}$ ed. Wiley Blackwell, Iowa. 1206 p.

Lillywhite H.B. \& Gatten Jr R.E. 1995. Physiology and functional anatomy, p.5-25. In: Warwick C., Frye F.L. \& Murphy J.B. (Eds), Health and Welfare of Captive Reptiles. Chapman and Hall, London.

López-Olvera J.L., Montané J., Marco I., Martínez-Silvestre A., Soler J. \& Lavin S. 2003. Effect of venipuncture site on hematologic and serum biochemical parameters in marginated tortoise (Testudo marginata). J. Wildl. Dis. 39(4):830-836.

Murrey M.J. 2006. Cardiopulmonary anatomy and physiology, p.127-129. In: Mader D.R. (Ed.), Reptile Medicine and Surgery. Saunders-Elsevier, St Louis.

Murrey M.J. 2000. Reptile blood sampling and artifact considerations, p.185-192. In: Fudge A.M. (Ed.), Laboratory Medicine Avian and Exotic Pets. W.B. Saunders, Philadelphia.

Paraná 2004. Instituto Tecnológico SIMEPAR. Disponível em <http:// www.simepar.br>Acesso em 30 out. 2004.

Raskin R.E. 2000. Reptilian complete blood count, p.193. In: Fudge A.M. (Ed.), Laboratory Medicine Avian and Exotic Pets. W.B. Saunders, Philadelphia.

Samour H.J., Risley D., March T., Savage B., Nieva O. \& Jones D.M. 1984. Blood sampling techniques in reptiles. Vet. Rec. 114(19):472-476.

Sykes J.M. \& Klaphake E. 2008. Reptile hematology. Vet. Clin. Exotic Pet Pract. 11:481-500. 